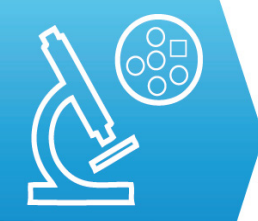

PATHOLOGY
1) Department Pathology, NRS Medical College, Kolkata, West Bengal, India

2) Department Tropical Medicine, Medical College, Kolkata, West Bengal, India
DOI: $10.15386 / \mathrm{mpr}-1951$

Manuscript received: 01.11.2020

Received in revised form: 27.12 .2020

Accepted: 17.01.2021

Address for correspondence:

mkgupta.pintu@gmail.com

This work is licensed under a Creative Commons Attribution-NonCommercialNoDerivatives 4.0 International License

\section{Immunohistochemical expression of MGMT in gliomas and its role in ascertaining patient survival}

Shristi Butta ${ }^{1}$, Manoj Kumar Gupta ${ }^{2}$

\begin{abstract}
Introduction. MGMT (O-6-methylguanine-DNA methyl transferase) is a DNA repair enzyme with implications on chemoresistance and subsequent patient prognosis. This study investigated the association of MGMT with the various grades and subtypes of gliomas and evaluated the associated clinical outcome of these patients.

Methods. This observational longitudinal follow up study spun over a period of 36 months and included 33 patients with primary glioma who underwent surgical interventions and chemoradiotherapy at a tertiary care center in Kolkata. The surgical samples were processed and histopathologically typed. Immunohistochemical analysis was done using anti- MGMT antibody and MGMT status was determined. Patients were followed up for 3 years.

Results. Males were 1.3 times more commonly affected by gliomas. Mean age was 42.9 years for females and 47.2 years for males. Frontal lobe was the most commonly involved site whereas focal neurological deficit was the most common symptom. Karnofsky performance score was higher for low grade gliomas and lower for high grade gliomas $(\mathrm{p}=0.04)$. Significant association was found between histopathological grade and MGMT immunoexpression $(\mathrm{p}=0.0001)$ as well as histopathological subtype and MGMT status $(\mathrm{p}=0.0036)$. On follow up, mean survival of the patients was 25.4 months. Significant association was found between MGMT status and survival of the patients $(\mathrm{p}=0.0437)$.

Conclusion. MGMT immunoexpression is significantly associated with different grades and subtypes of gliomas. In addition, MGMT has significant implications on chemoresistance and patient survival. Hence, MGMT expression should be mandatorily checked before starting the chemotherapy.
\end{abstract}

Keywords: MGMT, glioma, survival

\section{Introduction}

Gliomas constitute a vast majority of tumors intrinsic to the central nervous system [1]. These are treated primarily by complete surgical resection followed by radiotherapy and chemotherapy using alkylating agents like temozolomide [2]. However, the prognosis of these gliomas despite a range of therapies, remains dismal. The reason could be attributed to the resistance to chemotherapeutic agents like temozolomide (TMZ). One of the factors responsible for chemoresistance is O-6-methylguanineDNA methyl transferase (MGMT). Epigenetic silencing by hypermethylation of $\mathrm{CpG}$ rich islands (cytidine phosphateguanosine dinucleotide) of MGMT results in silencing of MGMT gene and loss of its immunoexpression. MGMT status has been considered as a good prognostic indicator for gliomas [3]. There are several techniques involved in the detection of the MGMT status like immunohistochemistry (IHC), methylation-specific PCR, pyrophosphate sequencing and real-time 
quantitative PCR. Among these detection techniques, IHC is one of the easiest, user friendly and cost - effective techniques. In the present study we aim to investigate the expression of MGMT in different histopathological grades and subtypes of gliomas and determine the role of MGMT in ascertaining the patient survival.

\section{Methods}

This observational, longitudinal follow up study was conducted in the department of Pathology of NRS medical college and hospital, Kolkata. The study included 33 patients with primary glioma admitted to the department of Neurosurgery, surgically treated and post-operatively received chemoradiotherapy. The study was conducted over a period of 36 months. Surgical samples of the patients were processed histopathologically, stained with Hematoxylin \& Eosin (H\&E) and graded microscopically. Subsequently gliomas were histopathologically typed according to the WHO standards. Immunohistochemistry was performed using mouse monoclonal anti-MGMT antibody. Endothelial cells were taken as positive internal control. Negation of primary antibody was taken as negative control. 5-micrometer thick sections of $10 \%$ formalin fixed paraffin embedded (FFPE) tissue were cut for immunohistochemistry (IHC). Deparaffinization was done using xylene and was subsequently treated with $0.3 \% \mathrm{H}_{2} \mathrm{O}_{2}$ in methanol for 20 minutes (hydrogen peroxide blocks the endogenous peroxidase activity and reduces background staining). The sections were then treated with Tris- Buffered Saline (TBS) plus 0.025\% Triton X-100 (this solution reduces the surface tension and allows all the reagents to mix properly in addition this also reduces non-specific binding). The sections were then incubated overnight with the primary antibody, mouse monoclonal anti-MGMT antibody (dilution of 1:50) (overnight incubation allows for more antibodies to bind and saturate its target). The slides were then incubated in enzyme-conjugate secondary antibody in TBS for 1 hour. Subsequently the slides were developed in DAB (deoxyaminobenzidine) chromogen for 10 minutes. Slides were rinsed in tap water and counterstained by hematoxylin. Sections were dehydrated, cleared and mounted. Nuclear expression of MGMT was considered positive. Scoring of MGMT was done as according to Mollemann et al [4], Negative: no positive tumor cells; Positive $+:<10 \%$ positive tumor cells; Positive $++: 10$ $50 \%$ positive tumor cells; Positive $+++:>50 \%$ positive tumor cells.

Subsequently, patients were regularly followed up by telephonic conversation or monthly hospital outdoor visits for ascertaining post - operative survival of the patient.

\section{Statistical analysis}

Statistical analysis was performed using Epi info TM version 7.2.4.0. Probability value or the $p$ value $<0.05$ was considered statistically significant.

\section{Results}

The mean age of females was 42.9 years whereas that of males was 47.2 years. Males were more prone to develop gliomas (57.58\%) as compared to females $(42.42 \%)$. However, Anova test revealed that there was no significant association was between age and gender of the patients $(p=0.53)$. Focal neurological deficit was the most common symptom (36.36\%) followed by headache (33.33\%). Karnofsky performance score (KPS) of 70 was most common (39.39\%) followed by a KPS of 60 (27.27\%). $45.45 \%$ of the cases belonged to WHO grade II followed by $33.33 \%$ which belonged to WHO grade IV. There was a significant association between KPS and WHO grade $(\mathrm{p}=0.04) .75 \%$ of WHO grade I gliomas had a KPS of 80 whereas $25 \%$ had a KPS of 90 . Among the WHO grade IV, $45.45 \%$ had a KPS of 70 and $36.36 \%$ had a KPS of 60 (Table I). Frontal lobe was the most commonly involved site $(33.33 \%)$ followed by parietal lobe $(21.21 \%)$. There was a significant association between tumor site and histopathological type of gliomas $(\mathrm{p}=0.01)$. Glioblastoma (33.3\%) accounted to be the most frequent histopathological type followed by diffuse astrocytoma (27.27\%). $45.45 \%$ cases showed $<10 \%$ positive staining of tumor cell nuclei (positive + ) for anti- MGMT antibody, $36.36 \%$ showed positive staining in $10-50 \%$ (positive++) of tumor cell nuclei, $12.12 \%$ cases showed positivity in $>50 \%$ tumor cell nuclei (positive +++ ) while only 2 cases $(6.06 \%)$ were negative for MGMT (Figure 1a-c). MGMT status showed significant association with WHO grade (probability value $=0.0001$ ) (Table II). All the cases of WHO grade IV, grade III and grade II glioma were MGMT positive whereas $50 \%$ cases of WHO grade I glioma were negative for MGMT. It was also found that $73.33 \%$ of grade II gliomas showed + positivity whereas $26.67 \%$ showed ++ positivity. All cases of grade III gliomas showed ++ positivity while $36.36 \%$ of grade IV gliomas revealed +++ positivity, $45.45 \%++$ positivity and $18.18 \%$ revealed + positivity (Table III). Different histopathological subtypes assessed were pilocytic astrocytoma (9.09\%), myxopapillary ependymoma $(3.03 \%)$, ependymoma $(9.09 \%)$, oligodendroglioma $(9.09 \%)$, diffuse astrocytoma (27.27\%), anaplastic astrocytoma (9.09\%) and glioblastoma (33.34\%) (Figure 2a-d). Statistically significant association was found between MGMT status and histopathological subtype of glioma $(\mathrm{p}=0.0036)$.

Subsequently, follow up of these patients revealed a mean survival of 25.4 months with a median of 24 months. Minimum survival was seen to be 12 months. As the study was restricted to 36 months, maximum survival was taken to be 36 months. A statistically significant correlation was found between MGMT status and survival of the patients $(\mathrm{p}=0.0437)$ (Table IV). 
Table I. Showing the distribution of Karnofsky Performance score (KPS) of patients and the WHO grade of gliomas.

\begin{tabular}{|c|c|c|c|c|c|}
\hline$\%$ & \multicolumn{4}{|c|}{ WHO GRADE } & $\%$ \\
\hline KPS & I & II & III & IV & Total \\
\hline 50 & 0 & 1 & 0 & 2 & 3 \\
\hline Row\% & $0.00 \%$ & $33.33 \%$ & $0.00 \%$ & $66.67 \%$ & $100.00 \%$ \\
\hline $\mathrm{Col} \%$ & $0.00 \%$ & $6.67 \%$ & $0.00 \%$ & $18.18 \%$ & $9.09 \%$ \\
\hline 60 & 0 & 5 & 0 & 4 & 9 \\
\hline Row \% & $0.00 \%$ & $55.56 \%$ & $0.00 \%$ & $44.44 \%$ & $100.00 \%$ \\
\hline $\mathrm{Col} \%$ & $0.00 \%$ & $33.33 \%$ & $0.00 \%$ & $36.36 \%$ & $27.27 \%$ \\
\hline 70 & 0 & 6 & 2 & 5 & 13 \\
\hline Row \% & $0.00 \%$ & $46.15 \%$ & $15.38 \%$ & $38.46 \%$ & $100.00 \%$ \\
\hline $\mathrm{Col} \%$ & $0.00 \%$ & $40.00 \%$ & $66.67 \%$ & $45.45 \%$ & $39.39 \%$ \\
\hline 80 & 3 & 3 & 1 & 0 & 7 \\
\hline Row\% & $42.86 \%$ & $42.86 \%$ & $14.29 \%$ & $0.00 \%$ & $100.00 \%$ \\
\hline $\mathrm{Col} \%$ & $75.00 \%$ & $20.00 \%$ & $33.33 \%$ & $0.00 \%$ & $21.21 \%$ \\
\hline 90 & 1 & 0 & 0 & 0 & 1 \\
\hline Row \% & $100.00 \%$ & $0.00 \%$ & $0.00 \%$ & $0.00 \%$ & $100.00 \%$ \\
\hline $\mathrm{Col} \%$ & $25.00 \%$ & $0.00 \%$ & $0.00 \%$ & $0.00 \%$ & $3.03 \%$ \\
\hline TOTAL & 4 & 15 & 3 & 11 & 33 \\
\hline Row\% & $12.12 \%$ & $45.45 \%$ & $9.09 \%$ & $33.33 \%$ & $100.00 \%$ \\
\hline $\mathrm{Col} \%$ & $100.00 \%$ & $100.00 \%$ & $100.00 \%$ & $100.00 \%$ & $100.00 \%$ \\
\hline
\end{tabular}

\begin{tabular}{c|c|c|} 
Chi-Squared & df & Probability \\
\hline 21.6811 & 12 & 0.0413
\end{tabular}
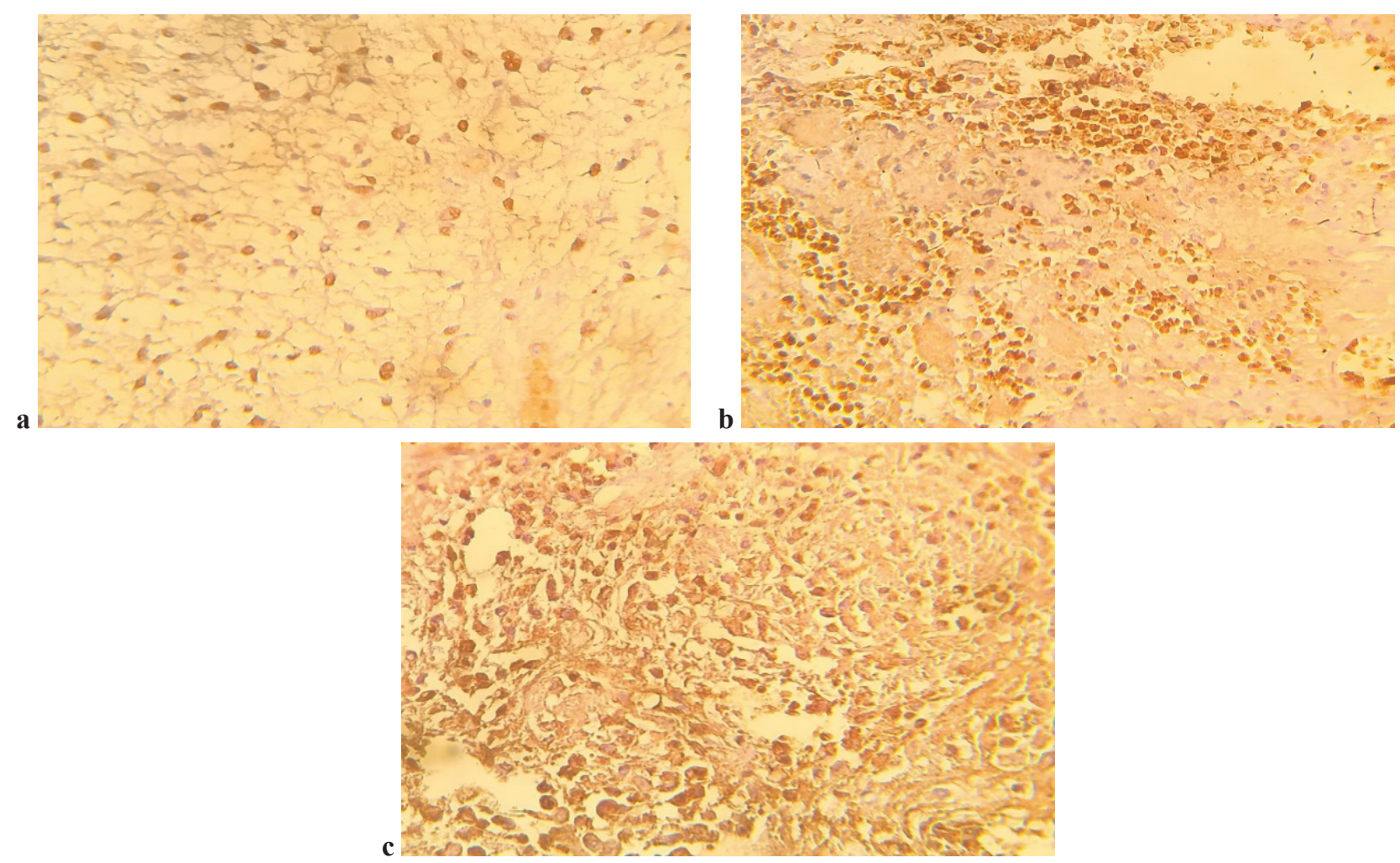

Figure 1. MGMT immunoexpression in Gliomas; 1a: MGMT $(+)<10 \%$ Cells with positive nuclear staining (400X); 1b: MGMT (++) $10-50 \%$ Cells with positive nuclear staining(100X); $1 \mathrm{c}$ : MGMT $(+++)>50 \%$ Cells with positive nuclear staining $(400 \mathrm{X})$. 
Table II. Showing the frequency of MGMT immunoexpression.

\begin{tabular}{lc|c} 
MGMT & Frequency & Percent \\
\hline NEGATIVE & 2 & $6.06 \%$ \\
POSITIVE+ & 15 & $45.45 \%$ \\
POSITIVE++ & 12 & $36.36 \%$ \\
POSITIVE+++ & 4 & $12.12 \%$ \\
Total & 33 & $100.00 \%$
\end{tabular}

Table III. Showing the distribution of MGMT and WHO grade of gliomas.

\begin{tabular}{|c|c|c|c|c|c|}
\hline$\%$ & \multicolumn{4}{|c|}{ WHO GRADE } & $\%$ \\
\hline MGMT & $\mathbf{I}$ & II & III & IV & Total \\
\hline NEGATIVE & 2 & 0 & 0 & 0 & 2 \\
\hline Row $\%$ & $100.00 \%$ & $0.00 \%$ & $0.00 \%$ & $0.00 \%$ & $100.00 \%$ \\
\hline $\mathrm{Col} \%$ & $50.00 \%$ & $0.00 \%$ & $0.00 \%$ & $0.00 \%$ & $6.06 \%$ \\
\hline POSITIVE+ & 2 & 11 & 0 & 2 & 15 \\
\hline Row\% & $13.33 \%$ & $73.33 \%$ & $0.00 \%$ & $13.33 \%$ & $100.00 \%$ \\
\hline $\mathrm{Col} \%$ & $50.00 \%$ & $73.33 \%$ & $0.00 \%$ & $18.18 \%$ & $45.45 \%$ \\
\hline POSITIVE++ & 0 & 4 & 3 & 5 & 12 \\
\hline Row $\%$ & $0.00 \%$ & $33.33 \%$ & $25.00 \%$ & $41.67 \%$ & $100.00 \%$ \\
\hline $\mathrm{Col} \%$ & $0.00 \%$ & $26.67 \%$ & $100.00 \%$ & $45.45 \%$ & $36.36 \%$ \\
\hline POSITIVE+++ & 0 & 0 & 0 & 4 & 4 \\
\hline Row $\%$ & $0.00 \%$ & $0.00 \%$ & $0.00 \%$ & $100.00 \%$ & $100.00 \%$ \\
\hline $\mathrm{Col} \%$ & $0.00 \%$ & $0.00 \%$ & $0.00 \%$ & $36.36 \%$ & $12.12 \%$ \\
\hline TOTAL & 4 & 15 & 3 & 11 & 33 \\
\hline Row\% & $12.12 \%$ & $45.45 \%$ & $9.09 \%$ & $33.33 \%$ & $100.00 \%$ \\
\hline $\mathrm{Col} \%$ & $100.00 \%$ & $100.00 \%$ & $100.00 \%$ & $100.00 \%$ & $100.00 \%$ \\
\hline Chi-Squared & $\mathrm{df}$ & bability & & & \\
\hline
\end{tabular}
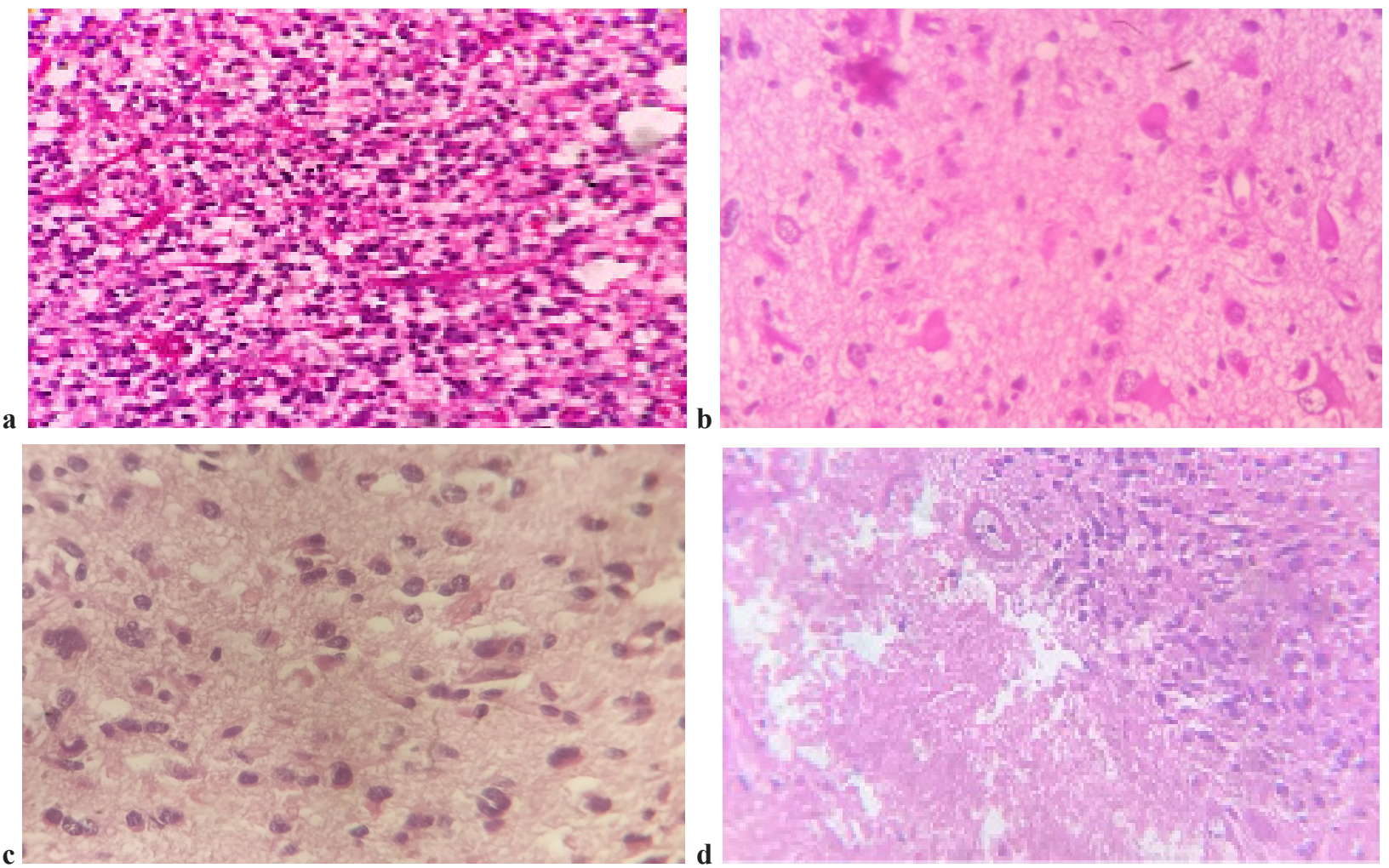

Figure 2. Histopathological subtypes of gliomas; 2a: Oligodendroglioma (WHO grade II) with characteristic fried egg appearance of cells and chicken wire blood vessels (H\&E, 100X); 2b: Gemistocytic astrocytoma (WHO grade II) with neoplastic gemistocytes ( H\&E, 400X); 2c: Diffuse astrocytoma (WHO grade II) with characteristic ependymal rosettes and perivascular pseudorosettes (H\&E, 400X); 2d: Glioblastoma (WHO grade IV) with high grade nuclear atypia and wide area of necrosis(H\&E, 400X). 
Table IV. Showing the distribution of MGMT and patient survival (in months).

\begin{tabular}{|c|c|c|c|c|c|c|c|c|c|c|c|c|c|c|}
\hline \multirow[b]{2}{*}{ MGMT } & \multicolumn{13}{|c|}{ SURVIVAL IN MONTHS } & \multirow[b]{2}{*}{ Total } \\
\hline & 12 & 14 & 15 & 16 & 18 & 22 & 23 & 24 & 26 & 28 & 30 & 32 & 36 & \\
\hline NEGATIVE & 0 & 0 & 0 & 0 & 0 & 0 & 0 & 0 & 0 & 0 & 0 & 0 & 2 & 2 \\
\hline Row\% & $0.00 \%$ & $0.00 \%$ & $0.00 \%$ & $0.00 \%$ & $0.00 \%$ & $0.00 \%$ & $0.00 \%$ & $0.00 \%$ & $0.00 \%$ & $0.00 \%$ & $0.00 \%$ & $0.00 \%$ & $100.00 \%$ & $100.00 \%$ \\
\hline $\mathrm{Col} \%$ & $0.00 \%$ & $0.00 \%$ & $0.00 \%$ & $0.00 \%$ & $0.00 \%$ & $0.00 \%$ & $0.00 \%$ & $0.00 \%$ & $0.00 \%$ & $0.00 \%$ & $0.00 \%$ & $0.00 \%$ & $22.22 \%$ & $6.06 \%$ \\
\hline POSITIVE+ & 0 & 0 & 0 & 0 & 0 & 1 & 0 & 2 & 1 & 1 & 2 & 1 & 7 & 15 \\
\hline Row\% & $0.00 \%$ & $0.00 \%$ & $0.00 \%$ & $0.00 \%$ & $0.00 \%$ & $6.67 \%$ & $0.00 \%$ & $13.33 \%$ & $6.67 \%$ & $6.67 \%$ & $13.33 \%$ & $6.67 \%$ & $46.67 \%$ & $100.00 \%$ \\
\hline $\mathrm{Col} \%$ & $0.00 \%$ & $0.00 \%$ & $0.00 \%$ & $0.00 \%$ & $0.00 \%$ & $50.00 \%$ & $0.00 \%$ & $40.00 \%$ & $100.00 \%$ & $50.00 \%$ & $100.00 \%$ & $100.00 \%$ & $77.78 \%$ & $45.45 \%$ \\
\hline POSITIVE++ & 0 & 2 & 0 & 2 & 2 & 1 & 1 & 3 & 0 & 1 & 0 & 0 & 0 & 12 \\
\hline Row\% & $0.00 \%$ & $16.67 \%$ & $0.00 \%$ & $16.67 \%$ & $16.67 \%$ & $8.33 \%$ & $8.33 \%$ & $25.00 \%$ & $0.00 \%$ & $8.33 \%$ & $0.00 \%$ & $0.00 \%$ & $0.00 \%$ & $100.00 \%$ \\
\hline $\mathrm{Col} \%$ & $0.00 \%$ & $100.00 \%$ & $0.00 \%$ & $100.00 \%$ & $66.67 \%$ & $50.00 \%$ & $100.00 \%$ & $60.00 \%$ & $0.00 \%$ & $50.00 \%$ & $0.00 \%$ & $0.00 \%$ & $0.00 \%$ & $36.36 \%$ \\
\hline POSITIVE+++ & 1 & 0 & 2 & 0 & 1 & 0 & 0 & 0 & 0 & 0 & 0 & 0 & 0 & 4 \\
\hline Row\% & $25.00 \%$ & $0.00 \%$ & $50.00 \%$ & $0.00 \%$ & $25.00 \%$ & $0.00 \%$ & $0.00 \%$ & $0.00 \%$ & $0.00 \%$ & $0.00 \%$ & $0.00 \%$ & $0.00 \%$ & $0.00 \%$ & $100.00 \%$ \\
\hline $\mathrm{Col} \%$ & $100.00 \%$ & $0.00 \%$ & $100.00 \%$ & $0.00 \%$ & $33.33 \%$ & $0.00 \%$ & $0.00 \%$ & $0.00 \%$ & $0.00 \%$ & $0.00 \%$ & $0.00 \%$ & $0.00 \%$ & $0.00 \%$ & $12.12 \%$ \\
\hline TOTAL & 1 & 2 & 2 & 2 & 3 & 2 & 1 & 5 & 1 & 2 & 2 & 1 & 9 & 33 \\
\hline Row\% & $3.03 \%$ & $6.06 \%$ & $6.06 \%$ & $6.06 \%$ & $9.09 \%$ & $6.06 \%$ & $3.03 \%$ & $15.15 \%$ & $3.03 \%$ & $6.06 \%$ & $6.06 \%$ & $3.03 \%$ & $27.27 \%$ & $100.00 \%$ \\
\hline $\mathrm{Col} \%$ & $100.00 \%$ & $00.00 \%$ & $100.00 \%$ & $100.00 \%$ & $100.00 \%$ & $100.00 \%$ & $100.00^{\circ}$ & $100.00^{\circ}$ & $100.00 \%$ & $00.00 \%$ & $100.00 \%$ & $100.00^{\circ}$ & $100.00^{\circ}$ & $100.00 \%$ \\
\hline
\end{tabular}

\section{Discussion}

Despite a range of treatment modalities such as surgery and chemo-radiotherapy the median survival of patients with high grade glioma is about a year or so. Chemoresistance has a significant impact on the therapeutic management of gliomas. O-6-methylguanineDNA methyltransferase (MGMT) is a DNA repair enzyme which antagonizes the effects of therapeutic agents like temozolomide and inhibits killing of tumor cells $[5,6]$.

This study found that MGMT status played an important role in ascertaining patient survival. Cases showing low to absent immunoexpression for MGMT had a better prognosis and overall survival as compared to those with moderate to high immunoexpression for MGMT. This could be attributed to the fact that MGMT counteracts the effects of the alkylating agents. Its decreased immunoexpression accounts for better chemotherapeutic response and increased overall survival. Temozolomide is a DNA alkylating agent which has the ability to alkylate the DNA at the O-6 position of guanine and hence results in death of tumor cells. MGMT gene resides on chromosome number 10q26. This gene encodes a protein which removes alkyl groups from O-6 position of guanine thus repairing the damaged DNA subjected to alkylation or rather temozolomide induced alkylation [7]. Hence, epigenetic silencing by promotor hypermethylation of $\mathrm{CpG}$ rich islands of MGMT results in loss of its immunoexpression and a better response to the temozolomide chemotherapy. In addition, MGMT immunoexpression was found to be significantly associated with WHO grade and histopathological subtype of gliomas with high grade gliomas showing a stronger expression for MGMT as compared to low grade gliomas. This further explains the reason behind the chemo-resistance in high grade gliomas and its subsequent recurrence and adverse prognosis while lower grade gliomas are usually more chemo responsive and have comparatively a better prognosis [8].

Regarding the epidemiological parameters studied, it was found that males were slightly more prone to develop gliomas as compared to females. This predominance of gliomas among males has been previously documented by several authors as well. This presumably shows that gender affects brain tumor pathogenesis. Hormonal influence has been well documented in some brain tumors particularly meningiomas; however, in gliomas the disparity in occurence between males and females is low with slight male predominance [9-11]. Further, age provided a valuable hint into the diagnosis of several subtypes of gliomas with low grade gliomas (WHO grade I) and ependymomas being more likely to occur in children and young adults whereas higher grade gliomas (WHO grade II,III and IV) being more likely to occur in older adults and elderly. The age distribution of gliomas according to subtype and grade has also revealed consistent findings with several studies from the past [12,13]. Furthermore, unlike most brain tumors focal neurological deficit was found to be the most common symptom at presentation followed by headache. This finding too has corroborated well with previous studies $[14,15]$. This shows that symptoms at presentation might vary and depends on the tumour site within the brain parenchyma, size of the tumor and spread of the tumor. In addition, Karnofsky Performance Scale (KPS) score is a scoring system widely used to ascertain patient prognosis. In this study, pre-operative KPS was found to be lower in 
high grade gliomas and higher in low grade gliomas. KPS scoring being a tool for functional evaluation of the patients has shown that individuals with lower pre-operative KPS score had a worse outcome and a poor overall survival. This has also been documented in several studies in literature $[16,17]$.

Hence, loss of immunoexpression for MGMT revealed a high concordant association with better overall survival of the patients during follow up. Cases with negative to low immunoexpression for MGMT had a better overall survival than those with moderate to strong immunoreactivity. A number of studies in the past have also unraveled that MGMT is a relatively strong biomarker for ascertaining patient survival possibly because of its role in reverting the chemotherapeutic response of TMZ like alkylating agents [18-26].

Thus, we recommend that MGMT status must be evaluated in all patients with gliomas undergoing chemotherapy. Determining the MGMT immune profiling would allow for more personalized patient management and enable better patient outcome.

\section{Conclusion}

We concludethat MGMT is a potentially valuable marker for gliomas. It has a significant association with the histopathological grade and subtype of gliomas. In addition, it has a strong role in predicting tumor prognosis and patient survival. Chemotherapeutic resistance in patients with moderate to strong expression for MGMT has also been implicated. Thus, MGMT immunohistochemistry should be carried out as a preliminary step in all glioma patients before starting chemotherapy using alkylating agents.

\section{References}

1. Wesseling P, Capper D. WHO 2016 Classification of gliomas. Neuropathol Appl Neurobiol. 2018;44:139-150.

2. Hadziahmetovic M, Shirai K, Chakravarti A. Recent advancements in multimodality treatment of gliomas. Future Oncol. 2011;7:1169-1183.

3. Hegi ME, Diserens AC, Gorlia T, Hamou MF, de Tribolet $\mathrm{N}$, Weller M, et al. MGMT gene silencing and benefit from temozolomide in glioblastoma. N Engl J Med. 2005;352:9971003.

4. Möllemann M, Wolter M, Felsberg J, Collins VP, Reifenberger G. Frequent promoter hypermethylation and low expression of the MGMT gene in oligodendroglial tumors. Int J Cancer. 2005;113:379-385.

5. Friedman HS, Kerby T, Calvert H. Temozolomide and treatment of malignant glioma. Clin Cancer Res. 2000;6:2585-2597.

6. Weller M, Stupp R, Reifenberger G, Brandes AA, van den Bent MJ, Wick W, et al. MGMT promoter methylation in malignant gliomas: ready for personalized medicine? Nat
Rev Neurol. 2010;6:39-51.

7. Kitange GJ, Carlson BL, Schroeder MA, Grogan PT, Lamont $\mathrm{JD}$, Decker PA, et al. Induction of MGMT expression is associated with temozolomide resistance in glioblastoma xenografts. Neuro Oncol. 2009;11:281-291.

8. Sarkaria JN, Kitange GJ, James CD, Plummer R, Calvert H, Weller M, et al. Mechanisms of chemoresistance to alkylating agents in malignant glioma. Clin Cancer Res. 2008;14:29002908.

9. Sun T, Plutynski A, Ward S, Rubin JB. An integrative view on sex differences in brain tumors. Cell Mol Life Sci. 2015;72:3323-3342.

10. Sun T, Warrington NM, Rubin JB. Why does Jack, and not Jill, break his crown? Sex disparity in brain tumors. Biol Sex Differ. 2012;3:3.

11. Butta S, Gupta MK. Study of epidemiological aspects and hormone receptor status of meningiomas. International Journal of Research in Medical Sciences. 2020;8:2482-2486.

12. Li K, Lu D, Guo Y, Wang C, Liu X, Liu Y, et al. Trends and patterns of incidence of diffuse glioma in adults in the United States, 1973-2014. Cancer Med. 2018;7:5281-90.

13. Merchant TE, Pollack IF, Loeffler JS. Brain tumors across the age spectrum: biology, therapy, and late effects. Semin Radiat Oncol. 2010;20:58-66.

14. IJzerman-Korevaar M, Snijders TJ, de Graeff A, Teunissen SCCM, de Vos FYF. Prevalence of symptoms in glioma patients throughout the disease trajectory: a systematic review. J Neurooncol. 2018;140:485-496.

15. Posti JP, Bori M, Kauko T, Sankinen M, Nordberg J, Rahi M, et al. Presenting symptoms of glioma in adults. Acta Neurol Scand. 2015;131:88-93.

16. Chambless LB, Kistka HM, Parker SL, Hassam-Malani L, McGirt MJ, Thompson RC. The relative value of postoperative versus preoperative Karnofsky Performance Scale scores as a predictor of survival after surgical resection of glioblastoma multiforme. J Neurooncol. 2015;121:359364.

17. Oken MM, Creech RH, Tormey DC, Horton J, Davis TE, McFadden ET, et al. Toxicity and response criteria of the Eastern Cooperative Oncology Group. Am J Clin Oncol. 1982;5:649-655.

18. Rodriguez FJ, Thibodeau SN, Jenkins RB, Schowalter KV, Caron BL, O'neill BP, et al. MGMT immunohistochemical expression and promoter methylation in human glioblastoma. Appl Immunohistochem Mol Morphol. 2008;16:59-65.

19. Wang Y, Fan X, Zhang C, Zhang T, Peng X, Li S, et al. Anatomical specificity of O6-methylguanine DNA methyltransferase protein expression in glioblastomas. J Neurooncol. 2014;120:331-337.

20. Thon N, Eigenbrod S, Kreth S, Lutz J, Tonn JC, Kretzschmar $\mathrm{H}$, et al. IDH1 mutations in grade II astrocytomas are associated with unfavorable progression-free survival and prolonged postrecurrence survival. Cancer. 2012;118:452-460.

21. Belanich M, Pastor M, Randall T, Guerra D, Kibitel J, Alas $\mathrm{L}$, et al. Retrospective study of the correlation between the DNA repair protein alkyltransferase and survival of brain tumor patients treated with carmustine. Cancer Res. 
1996;56:783-788.

22. Silber JR, Blank A, Bobola MS, Ghatan S, Kolstoe DD, Berger MS. O6-methylguanine-DNA methyltransferasedeficient phenotype in human gliomas: frequency and time to tumor progression after alkylating agent-based chemotherapy. Clin Cancer Res.1999;5:807-814.

23. Gerson SL. MGMT: its role in cancer aetiology and cancer therapeutics. Nat Rev Cancer. 2004;4:296-307.

24. Paz MF, Yaya-Tur R, Rojas-Marcos I, Reynes G, Pollan M, Aguirre-Cruz L, et al. CpG island hypermethylation of the DNA repair enzyme methyltransferase predicts response to temozolomide in primary gliomas. Clin Cancer Res. 2004;10:4933-4938.

25. Weiler M, Hartmann C, Wiewrodt D, Herrlinger U, Gorlia $\mathrm{T}$, Bähr $\mathrm{O}$, et al. Chemoradiotherapy of newly diagnosed glioblastoma with intensifed temozolomide. Int $\mathrm{J}$ Radiat Oncol Biol Phys. 2010;77:670-676.

26. Weller M, Felsberg J, Hartmann C, Berger H, Steinbach JP, Schramm J, et al. Molecular predictors of progressionfree and overall survival in patients with newly diagnosed glioblastoma: a prospective translational study of the German Glioma Network. J Clin Oncol. 2009;27:5743-5750. 